\title{
Effets de la fertilisation des sols à l'aide des déchets ménagers solides compostés dans les décharges sur le rendement et la qualité chimique de la laitue (Lactuca sativa L.)
}

\author{
Zoumana KONATE ${ }^{1 *}$, Hebert Damien Akré ABOBI ${ }^{1}$, Faustin Dago $\mathrm{SOKO}^{2}$ et \\ Albert YAO-KOUAME ${ }^{3}$
}

\author{
${ }^{1}$ Université Jean Lorougnon Guédé de Daloa / Unité de Formation et de Recherche en Agroforesterie. \\ Département : Agropédologie, Télédétection et SIG, BP 150 Daloa (Côte d'Ivoire) \\ ${ }^{2}$ Université Jean Lorougnon Guédé de Daloa / Unité de Formation et de Recherche en Agroforesterie. \\ Département : Physiologie Végétale, BP 150 Daloa (Côte d'Ivoire) \\ ${ }^{3}$ Université Félix Houphouët-Boigny, Unité de Formation et de Recherche en Sciences de la Terre et des \\ Ressources Minières (UFR-STRM), Département des Sciences du Sol, 22 BP 582 Abidjan 22, Côte d'Ivoire. \\ *Auteur correspondant ; E-mail: zoumko@yahoo.fr
}

\section{RESUME}

Dans les zones urbaines et périurbaines, les composts d'ordures ménagères sont souvent utilisés par les producteurs de maraîchers pour fertiliser les sols. La présente étude réalisée dans la ville de Daloa en Côte d'Ivoire avait pour but d'évaluer la valeur agronomique des déchets ménagers compostés à l'air libre ainsi que la qualité des productions de laitue (Lactuca sativa) obtenues sur des sols amendés avec ce type de compost. Pour ce faire, un dispositif en bloc de Fischer a permis de comparer les effets de l'apport au sol d'un compost de déchets ménagers à la dose appliquée régulièrement par les producteurs (20 t/ha) aux effets d'un apport au sol de l'engrais minéral NPK 12-22-22 à la dose de $250 \mathrm{~kg} / \mathrm{ha}$ et d'un témoin sans fertilisation du sol. Les résultats obtenus sur trois cycles de culture de la laitue ont révélé que les teneurs en matières organiques $(12,15$ $\%)$ et en azote $(0,65 \%)$ du compost utilisé sont relativement élevées comparativement à la norme AFNOR, d'où une valeur fertilisante assez intéressante de ce compost. Aussi, les teneurs en éléments traces métalliques toxiques, notamment le fer $\left(1,911 \mathrm{mg} \cdot \mathrm{kg}^{-1}\right)$ et le zinc $\left(1,55 \mathrm{mg} \cdot \mathrm{kg}^{-1}\right)$ trouvées dans ce compost sont-elles très faibles et inférieures aux seuils critiques définis dans les sols agricoles par la législation suisse. Cependant, l'usage répété des composts pouvant entraîner une accumulation de métaux lourds dans le sol, il convient pour les producteurs de fabriquer des composts à base d'ordures ménagères qui ont été préalablement triées de sorte à obtenir des composts de moins en moins pourvus en métaux lourds, et donc moins dangereux pour l'agriculture.

(C) 2018 International Formulae Group. All rights reserved.

Mots clés: Fertilisation, compost, rendement, qualité, laitue.

\section{Effects of soil fertilization using composted solid household waste in landfills on yield and chemical quality of lettuce (Lactuca sativa $\mathbf{L}$.)}

\begin{abstract}
In urban and peri-urban areas, garbage compost is often used by market gardeners to fertilize soils. The present study conducted in the city of Daloa in Côte d'Ivoire aimed to evaluate the agronomic value of
\end{abstract}


composted household waste in the open air and the quality of lettuce (Lactuca sativa) produced on soils amended with this type of compost. To do this, a Randomized Complete Block Design (RCBD) with three replicates made possible to compare the effects of the soil intake of a domestic compost with the rate regularly applied by the growers ( $20 \mathrm{t} / \mathrm{ha}$ ) to the effects of a contribution to the NPK 12-22-22 mineral fertilizer at a rate of $250 \mathrm{~kg} / \mathrm{ha}$ and a control without soil fertilization. The results obtained revealed that the organic matter $(12,15 \%)$ and nitrogen $(0,65 \%)$ contents of the compost used are relatively high compared to the AFNOR standard, hence interesting fertilizing value of this compost. As a result, the levels of toxic metal trace elements, particularly iron $\left(1,911 \mathrm{mg} \cdot \mathrm{kg}^{-1}\right)$ and zinc $\left(1,55 \mathrm{mg} \cdot \mathrm{kg}^{-1}\right)$ found in this compost, are very low and below the thresholds defined in agricultural soils by Swiss legislation. However, as the repeated use of composts can lead to the accumulation of toxic heavy metals in the soil, it is appropriate for producers to produce composts based on household waste previously sorted so as to obtain composts less and less loaded with heavy metals.

(C) 2018 International Formulae Group. All rights reserved.

Keywords: Fertilization, compost, yield, quality, lettuce.

\section{INTRODUCTION}

Dans les pays en voie de développement, et particulièrement ceux de l'Afrique subsaharienne, l'agriculture urbaine joue un rôle important sur la sécurité alimentaire des villes (Bricas et Seck 2004 ; Lesafre 2004). L'approvisionnement en denrées alimentaires des villes et l'accès à des produits frais de proximité sont des atouts majeurs de cette agriculture auxquels les agrosystèmes maraîchers urbains et périurbains contribuent très fortement. $\mathrm{La}$ gestion de la matière organique dans les sols de ces agrosystèmes (Dragon et Icard 2010) revêt en général une importance capitale, non seulement, sur les plans agronomique et économique, mais aussi sur le plan environnemental (Houot 2005). En effet, les composts de déchets ménagers sont de plus en plus utilisés par les producteurs en remplacement des engrais chimiques, du fait des coûts élevés de ces derniers. Cependant, ces composts d'ordures ménagères peuvent conduire à une accumulation d'éléments métalliques dans le sol et ensuite dans le végétal (Djogbedé et al., 2012) surtout quand ils n'ont pas été obtenus suite à un tri sélectif des ordures, comme c'est le cas avec les composts d'ordures ménagères formés à l'air libre dans les décharges. Ces derniers étant souvent utilisés par les producteurs de maraîchers de la ville de Daloa (Zro et al., 2017b) peuvent constituer une source de pollution des sols et des productions par les éléments traces métalliques (Atidegla et al., 2011).

Ce constat amène à évaluer la valeur agronomique des déchets ménagers compostés à l'air libre ainsi que la qualité des productions obtenues sur les sols amendés avec ce type de compost.

La présente étude visait à évaluer les effets de ces déchets ménagers compostés à l'air libre sur quelques variables de croissance, de production et sur la qualité chimique de la laitue produite par les producteurs de maraîchers de la ville de Daloa.

\section{MATERIEL ET METHODES}

\section{Caractéristiques générales de la zone d'étude}

L'étude a été réalisée à Daloa entre $6^{\circ} 84$ et $6^{\circ} 91$ de latitude Nord et entre $6^{\circ} 41$ et $6^{\circ} 48$ de longitude Ouest (Figure 1), dans la région du Haut-Sassandra, située au Centreouest de la Côte d'Ivoire, pays de l'Afrique de l'ouest. Le régime climatique est celui du domaine Guinéen, caractérisé par un régime équatorial et subéquatorial. Les pluviométries moyennes sont comprises entre 1400 et 1600 mm par an. Le mois de juin représente le pic de la grande saison pluvieuse et celui de septembre, le pic de la petite saison des pluies. Ces deux maxima sont séparés par un ou deux mois plus ou moins pluvieux (Brou 2005). Selon Brou (2010), le paysage forestier de la zone d'étude varie progressivement de la forêt dense humide semi-décidue à une forêt défrichée mésophile. 
Le relief y est peu contrasté et peu varié, dominé par des plateaux de 200 à $400 \mathrm{~m}$ d'altitude (Avenard 1971). Les formations géologiques sont celles du Précambrien moyen, dominées essentiellement par les granites auxquels s'ajoutent quelques intrusions de schiste et de flysch (Avenard 1971 ; Dabbadie 1996) .

Les sols sont en général ferralitiques moyennement lessivés ou désaturés (Dabin et al., 1960). Cependant, le sol du site expérimental localisé au quartier Tazibouo de Daloa est un gleysol sableux (Zro et al., 2016).

Les activités économiques y sont assez diversifiées. Cependant, dans les villes de Côte d'Ivoire et particulièrement de Daloa, ce sont les cultures vivrières, les cultures maraîchères surtout qui jouent un rôle prépondérant.

\section{Matériel}

Pour cette étude, trois types essentiels de matériel ont été utilisés : un matériel végétal, un matériel fertilisant et un matériel technique.

Le matériel végétal était constitué de la laitue ou Lactuca sativa (Figure 2). Elle a été utilisée pour cette étude en raison de son fort ancrage dans les habitudes alimentaires des populations de la ville de Daloa et de son cycle de production court permettant en un temps relativement réduit, plusieurs séries d'observations et de mesures.

Le matériel fertilisant utilisé était constitué d'ordures ménagères solides compostées naturellement à l'air libre dans les décharges publiques de plus de 10 ans de la ville de Daloa (Figure 3 ) et l'engrais NPK 12 22-22 et les résidus de soja utilisés à la pépinière.

S'agissant du matériel technique utilisé, il était composé de plusieurs outils, notamment :

une balance Roberval, pour la mesure des quantités de matières de l'amendement apporté au sol sur le terrain ;

- une balance électronique de marque DENVER INSTRUMENT, pour les mesures de biomasses au laboratoire ;
- un mètre ruban, pour les mesures des planches sur la parcelle ;

- un appareil photo numérique, pour les prises de vue ;

- $\quad$ une machette, pour le défrichement de la parcelle ;

une pioche et une pelle, pour l'ouverture des fosses ;

un couteau de pédologue et des sachets plastiques, pour le prélèvement et la conservation des échantillons de sol.

\section{Méthodes}

Détermination de la valeur fertilisante des déchets ménagers compostés naturellement à l'air libre

La valeur fertilisante du compost d'ordures ménagères qui est son aptitude à fournir des éléments nutritifs $(\mathrm{N}, \mathrm{P}, \mathrm{K}, \mathrm{Ca}$, $\mathrm{Mg}, \mathrm{Na}$, etc.) et surtout l'azote ( $\mathrm{N})$ aux plantes a été déterminée à partir des compositions chimiques des échantillons de compost. Ainsi, la préparation du compost en vue des analyses au laboratoire a été faite selon la méthode de Chabalier et al. (2006) qui préconise l'échantillonnage, l'emballage et la conservation. L'emballage s'est fait dans des sachets plastiques ou des barquettes avec couvercle en matière plastique ou encore dans des flacons à large goulot avec couvercle, remplis aux deux tiers du volume. Le tout a été conservé au frais dans le réfrigérateur.

$\mathrm{Au}$ Laboratoire d'Analyse des Végétaux et des Sols (LAVESO) de l'École Supérieure d'Agronomie (ESA) sise à l'Institut National Polytechnique Félix Houphouët-Boigny, les échantillons de composts ont été séchés à $60^{\circ} \mathrm{C}$ à l'étuve pendant 48 heures, tamisés à $2 \mathrm{~mm}$ et broyés à $0,5 \mathrm{~mm}$.

Le $\mathrm{pH}$ du compost a été déterminé par la méthode électrométrique (LANO, 2008). L'azote total $(\mathrm{N})$, le carbone organique $(\mathrm{C})$, le phosphore $(\mathrm{P})$ et les bases échangeables $(\mathrm{K}$, $\mathrm{Mg}, \mathrm{Na}$ et $\mathrm{Ca}$ ) ont été dosés selon les techniques successives de la méthode d'attaque sulfochromique à froid, d'Olsen modifiée (LANO 2008) et de spectrophotométrie d'absorption atomique (LANO 2008). Pour évaluer les risques de 
pollution des sols, les teneurs en éléments traces métalliques (Fer, Plomb, zinc) ont été déterminées selon la méthode de la spectrométrie d'absorption atomique (Remon et al., 2009).

\section{Mise en place du dispositif expérimental}

Le plan d'expérimentation mis en place (Figure 4) a été établi selon le dispositif de Fischer (Dagnelie 2008). Chaque bloc était long de $10 \mathrm{~m}$ et constitué d'un ensemble de 3 parcelles élémentaires où des traitements différents (témoin, apport de NPK et apport de déchets ménagers) ont été répartis aléatoirement. La parcelle élémentaire était, quant à elle, une planche de $8 \mathrm{~m}^{2}$ (4 m x $\left.2 \mathrm{~m}\right)$. Une allée de $1 \mathrm{~m}$ de large a été observée autour de chaque bloc et par conséquent, autour de la parcelle entière.

\section{Conduite de l'essai}

\section{Mise en place et entretien de la pépinière}

La mise en place de la pépinière a consisté à un semis qui a été effectué à la volée sur des planches de $2 \mathrm{~m}$ de large et $4 \mathrm{~m}$ de long. La planche a été désinfectée avec du Diafuran $5 \mathrm{G}$ à raison de $50 \mathrm{~g} / \mathrm{m}^{2}$ et fertilisée avec $10 \mathrm{~kg} / \mathrm{m}^{2}$ de compost à base de résidus des récoltes de soja. C'est une pratique commune des producteurs de maraîchers de la ville de Daloa. Les planches ont été recouvertes avec des feuilles de palmier pendant les 3 premiers jours après le semis pour maintenir une humidité au niveau des jeunes plants repiqués puis recouvertes par une ombrière montée à une hauteur de $80 \mathrm{~cm}$ pour protéger les plants contre le soleil et les intempéries. Cet ombrage a été progressivement allégé en diminuant la quantité de feuilles puis complètement enlevé une semaine avant le repiquage des plants. La pépinière a été régulièrement arrosée (matin et soir) durant les 15 jours qu'a duré cette phase de l'étude.

\section{Repiquage et fertilisation de la laitue}

La pépinière a été abondamment arrosée avant d'arracher les plants âgés de 15 jours. Le repiquage a été effectué 15 jours après la mise en pépinière des plants. Le repiquage a été fait sur des planches de $8 \mathrm{~m}^{2}$ (4 m x $2 \mathrm{~m}$ ). Les plants ont été repiqués jusqu'au collet sur cinq lignes à raison de 0,30 $\mathrm{m}$ entre les lignes et $0,30 \mathrm{~m}$ sur la ligne, soit une densité de75000 plants/ha. Chaque parcelle élémentaire a été amendée :

- l'épandage des déchets ménagers à raison de 20t/ha, un mois avant le repiquage pour permettre sa décomposition et correspondant à la dose optimale utilisée par les producteurs de maraîchers ;

- $\quad$ apport de NPK de formule 12-22-22, à raison de $250 \mathrm{~kg} / \mathrm{ha}$ par parcelle élémentaire, en dose unique en engrais de fond, trois jours avant le repiquage des plants ;

- pas d'apport de fertilisant sur parcelles témoins.

\section{Traitements phytosanitaires et désherbage}

Aucun traitement phytosanitaire n'a été effectué pour éviter que ces traitements, qui sont à base de produits chimiques n'affectent les résultats attendus. Toutefois, un sarclage manuel a été régulièrement réalisé pour lutter contre l'enherbement.

\section{Mesures des variables de croissance et de production de la laitue}

Sur l'ensemble des douze parcelles élémentaires, les mesures ont été effectuées au centre de la parcelle en laissant les plants de bordure d'une manière identique, sur un total de 15 plants par traitement, en raison de 5 plants par ligne, suivant la diagonale (Figure 5). Ces mesures ont concerné, par traitement:

- le nombre de feuilles par plant

- $\quad$ et la surface foliaire. La surface foliaire (SF) a été calculée à partir de l'équation (Bonhomme et al., 1982): SF $\left(\mathrm{cm}^{2}\right)=\mathrm{L}$ $\mathrm{x} 1$ x 0,75 où: $\mathrm{SF}$ : la surface foliaire ; L (cm) : la longueur moyenne des feuilles et $1(\mathrm{~cm})$ : la largeur moyenne des feuilles ;

- la biomasse fraîche totale a concerné la masse de toute la plante de la laitue qui a été déterminée après arrachage et lavage de la plante pour débarrasser les racines de toute la masse de la terre.

Les observations ont débuté à la deuxième semaine après le repiquage par les mesures du nombre de feuilles et la SF. Ces mesures ont été répétées toutes les semaines 
suivantes jusqu'à la fin du cycle ( $6^{\text {ème }}$ semaine après le repiquage). La biomasse quant à elle, a été mesurée à la fin de chaque cycle de culture.

\section{Détermination de la qualité chimique des récoltes obtenues}

La qualité chimique de la laitue produite a été déterminée en mesurant sur des échantillons de récolte, les teneurs en éléments minéraux majeurs (azote, phosphore, calcium et magnésium) et en fer, zinc et plomb, qui sont des éléments traces métalliques (ETM). Les analyses ont été réalisées au Laboratoire d'Analyse des Végétaux et des Sols de l'Institut National Polytechnique Félix Houphouët-Boigny de Yamoussoukro. Pour ce faire, six plants entiers de laitue ont été prélevés à la fin de l'expérimentation, à raison de deux plants par traitement (Témoin, NPK et Compost de déchets ménagers). Ces deux plants correspondaient aux deux plus vigoureux du dernier cycle des cultures de chaque traitement. La méthode d'analyse employée est la technique de spectrométrie de masse (France-Ida, 1992). Les teneurs déterminées ont été comparées aux seuils existants en la matière (Mench et Baize, 2004 ; Lerot, 2006 ; Temgoua et al., 2015 ; FAO/OMS, 2016).

Analyse statistique des données

Les traitements statistiques des différentes données de l'étude ont été effectués à l'aide du logiciel STATISTICA 7.1. Les résultats obtenus ont été soumis à une analyse de variance au seuil de 5\% (Dagnelie 2008) pour apprécier l'effet des traitements sur les variables mesurées.

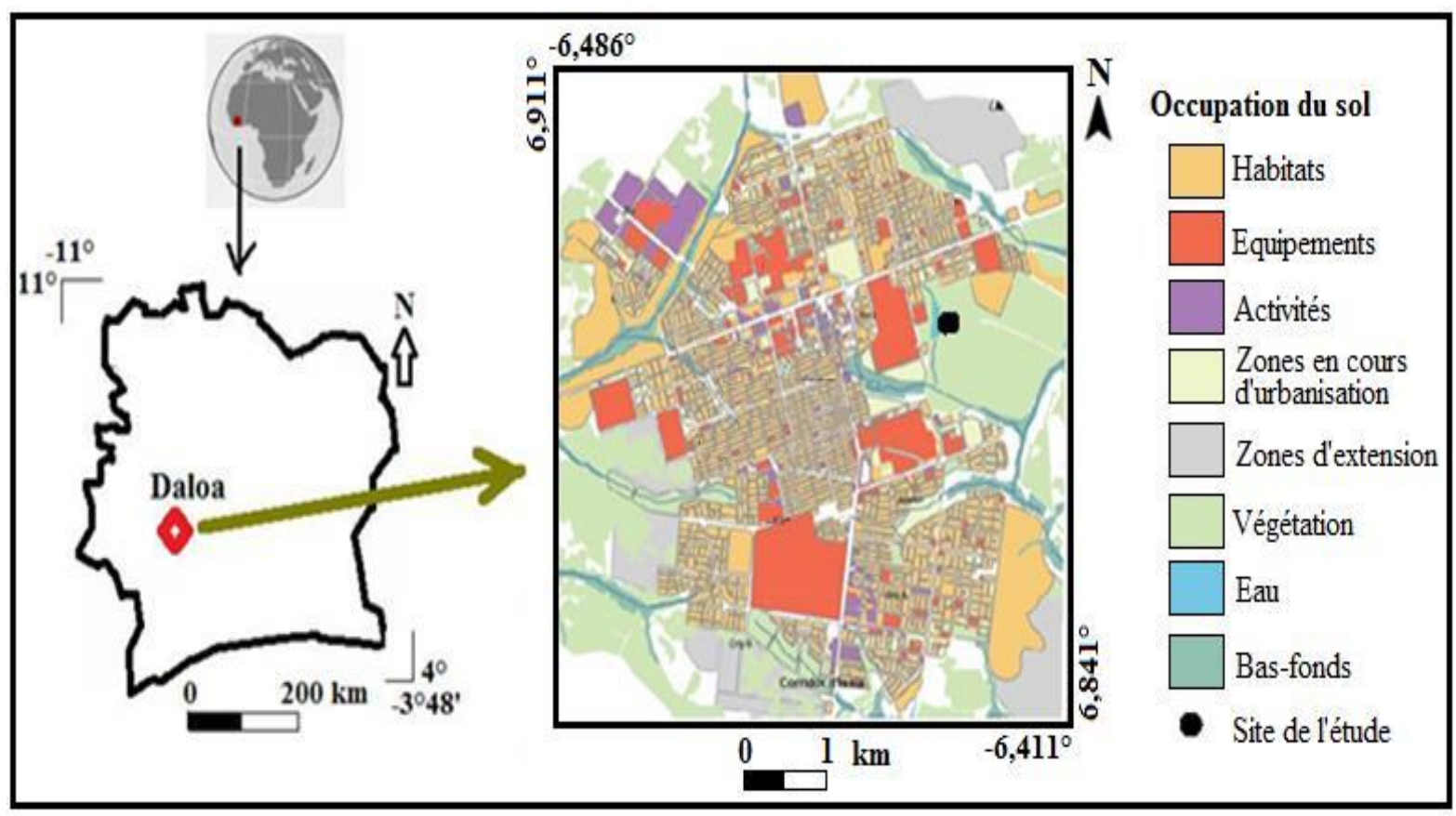

Figure 1 : Carte de localisation de la zone et du site de l'étude. 


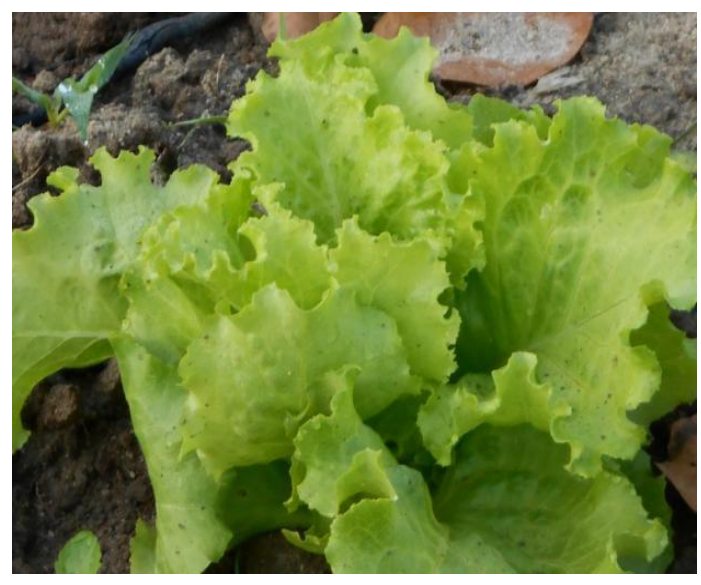

Figure 2 : Plant de laitue (Lactuca sativa L.) de 21 jours.

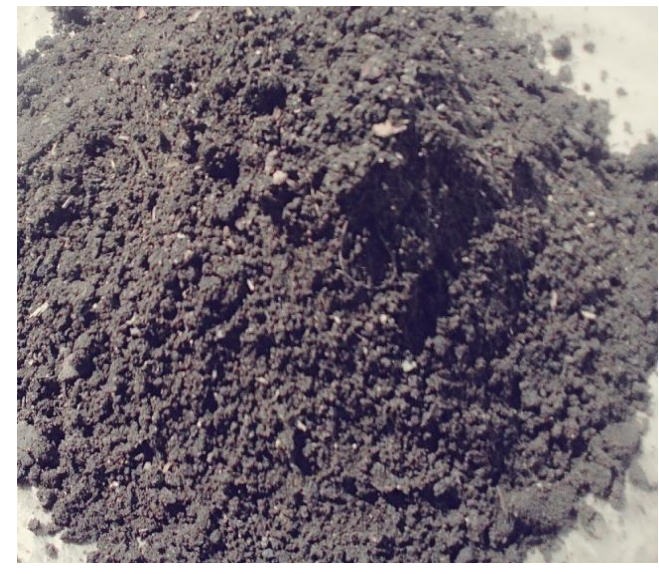

Figure 3 : Tas de compost d'ordures ménagères formés à l'air libre.

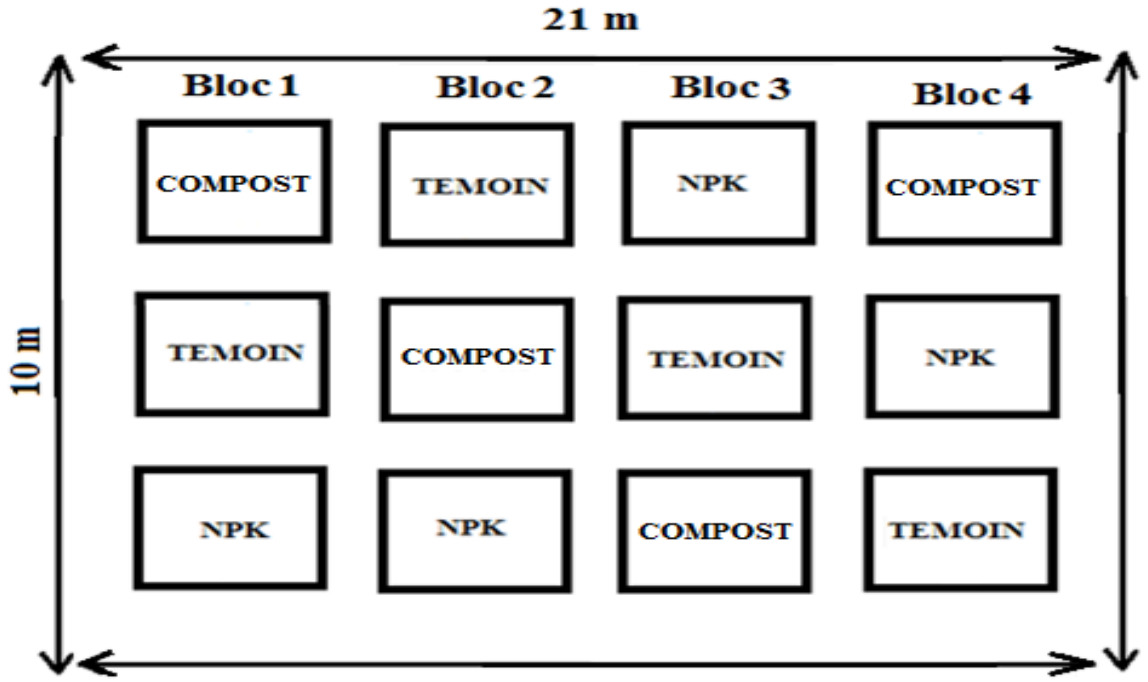

Figure 4 : Dispositif expérimental de l'étude.

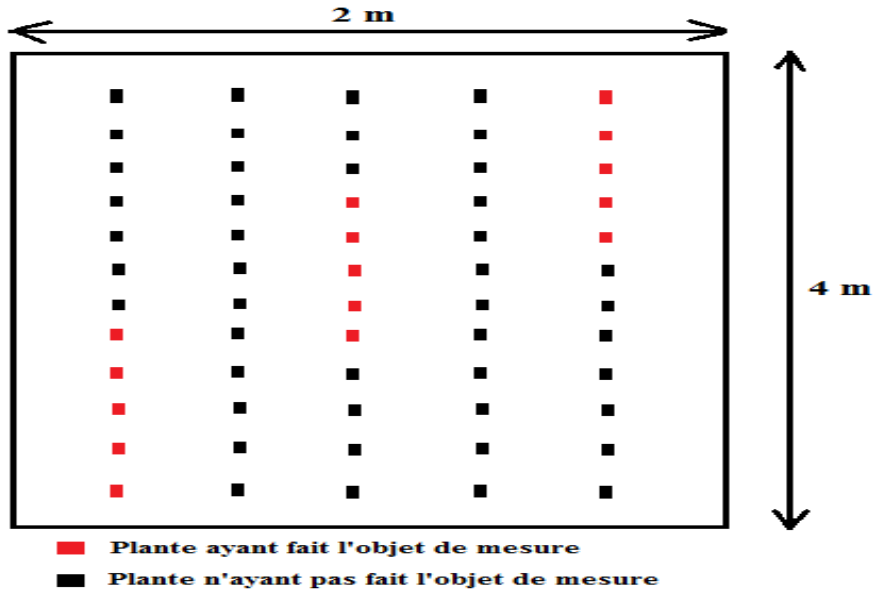

Figure 5: Schéma des plants observés par traitement 


\section{RESULTATS}

\section{Propriétés chimiques et organiques du compost de déchets ménagers}

Les résultats des différentes analyses réalisées au laboratoire sur le compost de déchets ménagers utilisés sont consignés dans les tableaux 1a et tableaux $1 \mathrm{~b}$ ci-dessous. Ces résultats ont montré que la valeur de la CEC est de $11,24 \mathrm{cmol} \cdot \mathrm{kg}^{-1}$. Le compost s'est avéré relativement basique $(\mathrm{pH}=7,3)$ et très bien pourvu en matières organiques $(12,15 \%)$. Les teneurs moyennes en éléments traces métalliques (ETM) ont été de 2,66 g. $\mathrm{kg}^{-1}$ pour le fer, $0,001 \mathrm{~g} \cdot \mathrm{kg}^{-1}$ pour le plomb et $0,42 \mathrm{~g} \cdot \mathrm{kg}^{-}$ ${ }^{1}$ pour le zinc. En effet, les teneurs trouvées dans ce compost ont été de 2,661 g.kg- ${ }^{1}, 0,42$ g.kg- ${ }^{1}$ et 0,001 g.kg- ${ }^{1}$, respectivement pour le fer, le zinc et le plomb.

\section{Variables de croissance et de production des plants de laitue par traitement Surface foliaire}

Quel que soit le traitement, les surfaces foliaires (Figure 6) se sont accrues avec le temps. Ainsi, des différences significatives ( $\mathrm{p}$ $<0,05)$ ont été observées entre les traitements à partir de la deuxième semaine du premier cycle des cultures et durant toutes les semaines des autres cycles.

\section{Nombre de feuilles}

Les différences observées entre les traitements n'ont pas été significatives au premier cycle de culture (semaine 1) concernant le nombre de feuilles de laitue lorsque les sols ont été amendés avec le compost et le NPK. Cependant, au fil du temps, c'est-à-dire à partir de la deuxième semaine, les différences commençaient à s'observer lorsque ces sols sont amendés avec le compost et le NPK (Figure 7). Cependant, le NPK a induit une légère croissance que le compost. Au second cycle, les différences entre les traitements ont été significatives durant tout le cycle. Cependant, les nombres moyens de feuilles les plus élevés ont été enregistré avec le traitement à base de compost. Concernant le troisième cycle, c'est à partir de la deuxième semaine que les différences entre les traitements sont apparues significatives. Aux semaines 2 et 3, le traitement à base du compost a permis d'enregistrer les nombres moyens de feuilles les plus élevés par rapport au traitement à base du NPK. Par contre, à la dernière semaine, c'est le traitement à base de compost qui a enregistré le plus faible nombre moyen de feuilles.

\section{Biomasses produites}

$\mathrm{Au}$ cours du cycle 1 (Figure 8), les masses moyennes les plus élevées concernant les biomasses épigées et les biomasses racinaires ont été enregistrées avec le traitement à base de l'engrais minéral NPK et les plus faibles par le traitement à base de compost. Les masses moyennes intermédiaires ont été obtenues par le traitement témoin. La tendance a été la même au niveau de l'évolution des biomasses totales (Figure 8).

L'évolution des biomasses, au cours du cycle 2, a été différente de celle observée au cycle 1, tant au niveau des biomasses totales $(110 \mathrm{~kg} / \mathrm{ha}$ avec les déchets ménagers, $59 \mathrm{~kg} / \mathrm{ha}$ avec le NPK et $38 \mathrm{~kg} / \mathrm{ha}$ avec le témoin) qu'au niveau des biomasses épigées $(99 \mathrm{~kg} / \mathrm{ha}$ avec les déchets ménagers, $55 \mathrm{~kg} / \mathrm{ha}$ avec le NPK et $35 \mathrm{~kg} / \mathrm{ha}$ avec le témoin) et racinaires (15 kg/ha avec les déchets ménagers, $10 \mathrm{~kg} / \mathrm{ha}$ avec le NPK et 5 $\mathrm{kg} / \mathrm{ha}$ avec le témoin). Les valeurs moyennes les plus élevées ont été enregistrées avec le traitement à base de compost par rapport au traitement à base de l'engrais minéral NPK. Par contre, pour le cycle 3, les tendances observées ont été similaires à celles du cycle précédent.

\section{Propriétés chimiques des récoltes}

Il n'y a pas de différence significative entre les traitements, concernant les teneurs des récoltes en phosphore, magnésium et calcium (Figure 9). Par contre, les teneurs en azote mesurées dans les récoltes issues du traitement à base de compost $\left(2,040 \mathrm{~g} \cdot \mathrm{kg}^{-1}\right)$ et $\mathrm{du}$ témoin $\left(1,88 \quad \mathrm{~g} . \mathrm{kg}^{-1}\right) \quad$ étaient significativement supérieures $(\mathrm{p}<0,05)$ à celles observées dans les récoltes issues du traitement NPK (1,40 g.kg- $\left.{ }^{1}\right)$. De même, la teneur en potassium dans les récoltes issues du traitement à base de compost $\left(4,717\right.$ g.kg- $\left.{ }^{1}\right)$ est apparue significativement supérieure ( $\mathrm{p}$ $<0,05)$ à celles dosées dans les récoltes issues 
des traitements NPK $\left(3,399\right.$ g.kg- $\left.{ }^{1}\right)$ et témoin $\left(3,819\right.$ g.kg- $\left.{ }^{1}\right)$.

Par ailleurs, la teneur en fer observée dans les récoltes provenant du traitement témoin $\left(2,144 \mathrm{mg} \cdot \mathrm{kg}^{-1}\right)$ est identique à celle des récoltes obtenues sur les sols fertilisés avec le NPK (2,171 mg.kg- $\left.{ }^{1}\right)$ (Figure 10).Ces deux traitements étaient ainsi différents du traitement à base de compost avec lequel l'on a enregistré $1,911 \mathrm{mg} . \mathrm{kg}-{ }^{1} \mathrm{de}$ fer dans les récoltes. Dans le cas du zinc, les différences observées entre les traitements sont similaires au cas du fer. Pour le plomb, quel que soit le traitement, il n'a pas été détecté dans les récoltes.

Tableau 1a: Propriétés chimiques et organiques ( $\mathrm{pH}$, matières organiques, phosphore assimilable) moyennes des déchets ménagers solides compostés à l'air libre.

\begin{tabular}{|c|c|c|c|c|c|}
\hline pH & & Matièr & organiques & & Phosphore \\
\hline Eau & $\mathrm{C}(\%)$ & $\mathrm{N}(\%)$ & $\mathrm{C} / \mathrm{N}$ & MO (\%) & P.ass (g.kg- $\left.{ }^{1}\right)$ \\
\hline $7,3 \pm 0,1$ & $7,05 \pm 0,1$ & $0,65 \pm 0,03$ & $10,84 \pm 0,3$ & $12,15 \pm 0,5$ & $0,056 \pm 0,03$ \\
\hline
\end{tabular}

Tableau 1b: Propriétés chimiques (complexe adsorbant, éléments traces métalliques) moyennes des déchets ménagers solides compostés à l'air libre.

\begin{tabular}{|c|c|c|c|c|c|c|c|}
\hline \multicolumn{5}{|c|}{ Complexe adsorbant } & \multicolumn{3}{|c|}{ Eléments traces métalliques } \\
\hline$\left(\right.$ cmol.kg-1) $^{-1}$ & & & & & $\overline{(\text { g.kg-1) }}$ & & \\
\hline CEC & $\mathbf{C a}^{2+}$ & $\mathbf{M g}^{+}$ & $\mathbf{K}^{+}$ & $\mathbf{N a}^{+}$ & $\mathrm{Fe}^{2+}$ & $\mathbf{P b}^{2+}$ & $\mathbf{Z n}^{2+}$ \\
\hline $11,24 \pm 0,6$ & $8,77 \pm 04$ & $2,29 \pm 0,1$ & $0,90 \pm 0,07$ & $0,16 \pm 0$ & $2,66 \pm 0,1$ & $0,001 \pm 0$ & $0,42 \pm 0,02$ \\
\hline
\end{tabular}

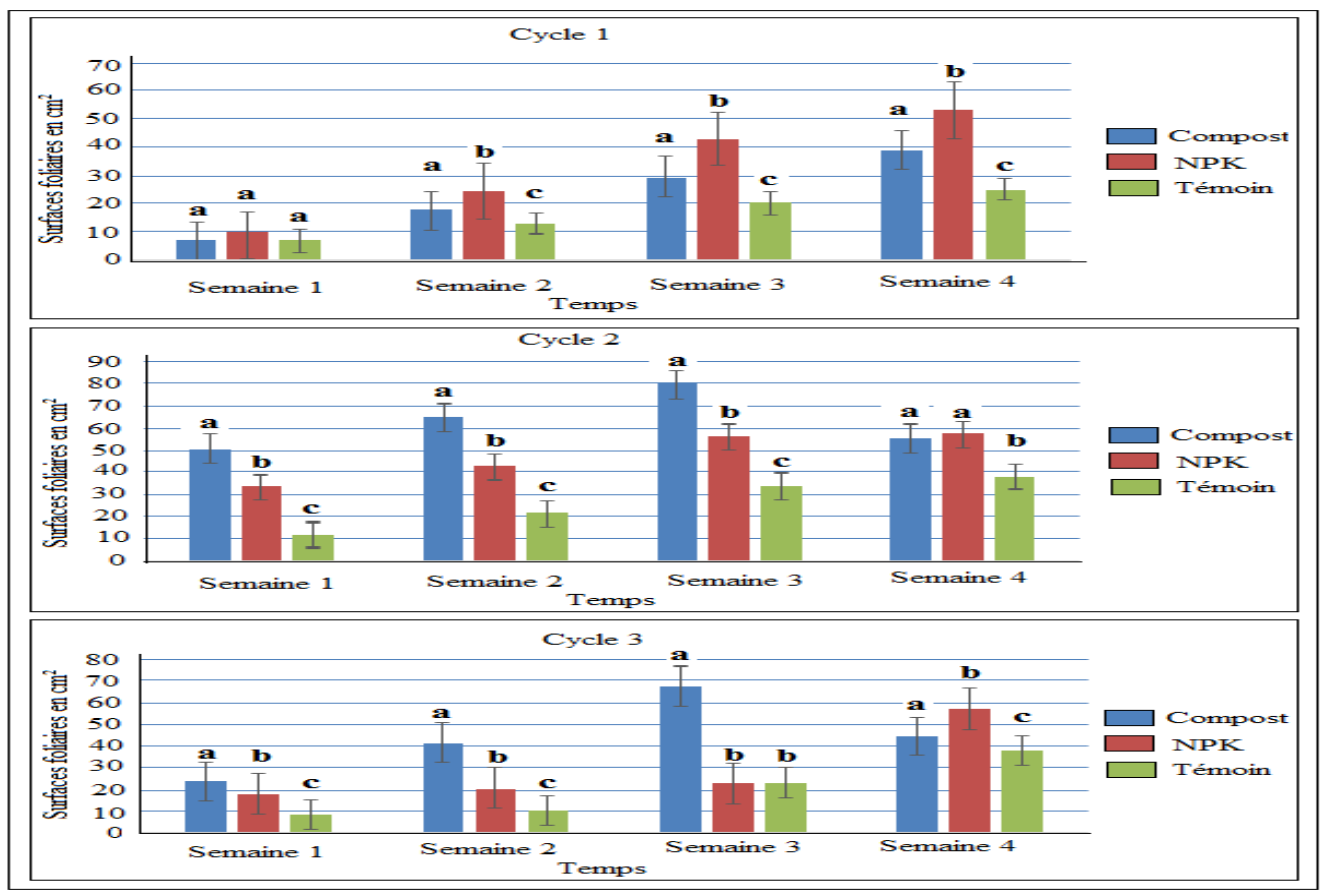

Les lettres $a$, $b$ et c désignent pour chaque traitement les valeurs moyennes significativement différentes au seuil de 5\%

Figure 6: Évolution de la surface foliaire de la laitue en fonction du temps et des trois modes de fertilisation. 


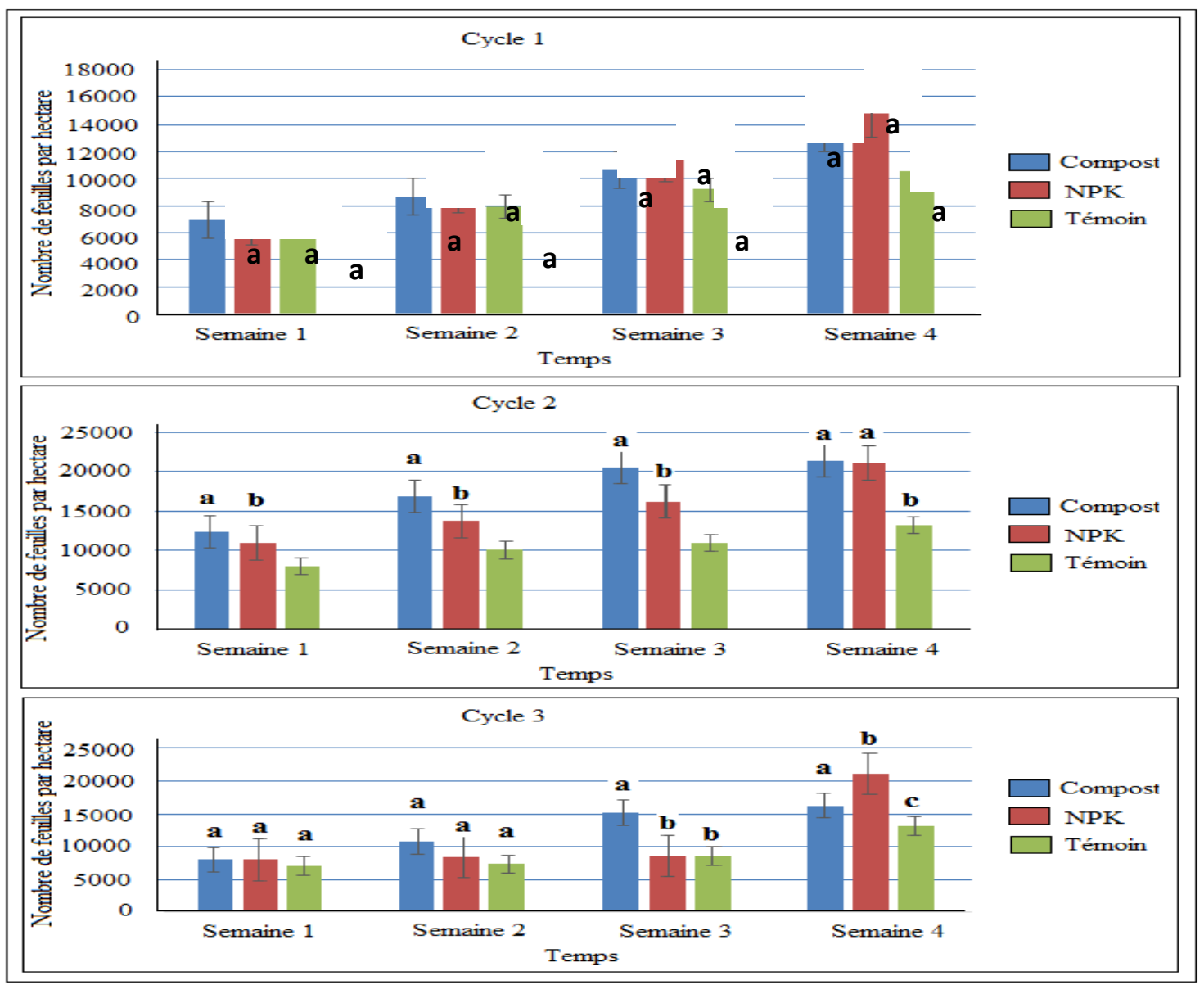

Les lettres $a, b$ et c désignent pour chaque traitement les valeurs moyennes significativement différentes au seuil de 5\%

Figure 7: Évolution du nombre de feuilles de la laitue en fonction du temps et des modes de fertilisation.

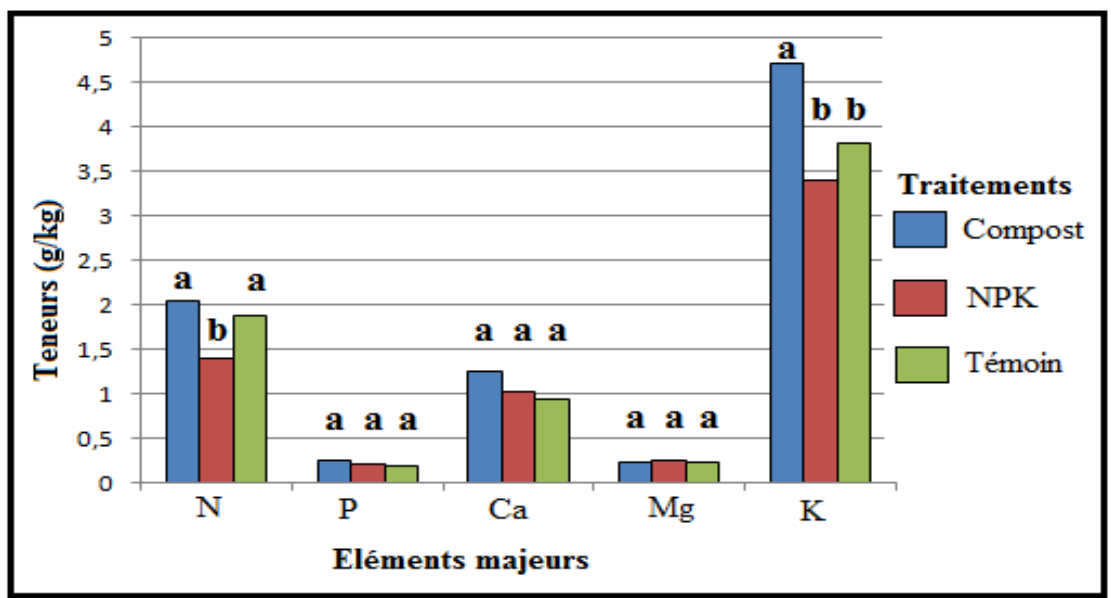

Les lettres a et b désignent pour chaque traitement les valeurs moyennes significativement différentes au seuil de 5\%

Figure 9: Teneurs en éléments minéraux majeurs et secondaires des récoltes de laitue en fonction des modes de fertilisation. 


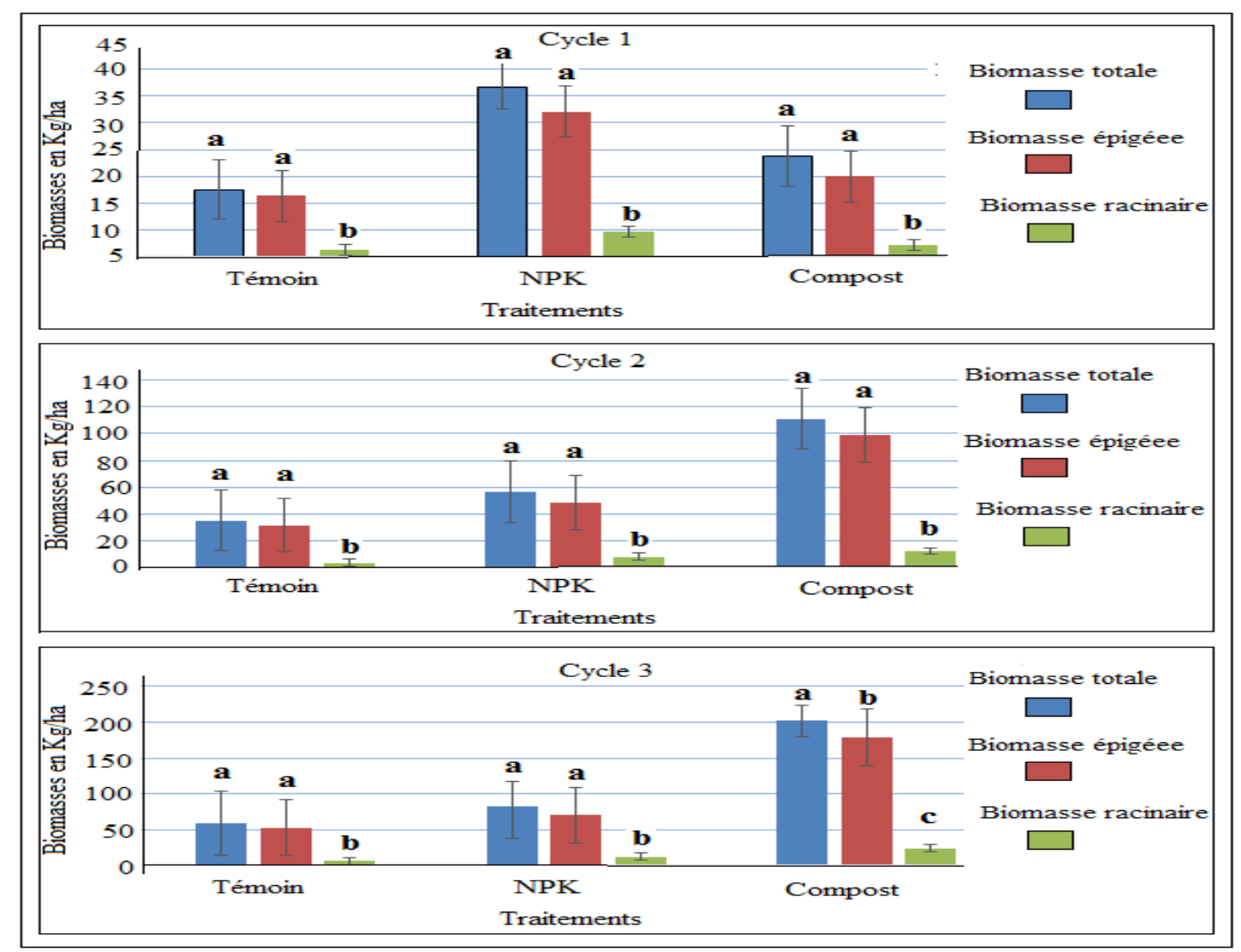

Les lettres $a$, b et c désignent pour chaque traitement les valeurs moyennes significativement différentes au seuil de $5 \%$

Figure 8 : Biomasse de laitue produite en fonction des modes de fertilisation.

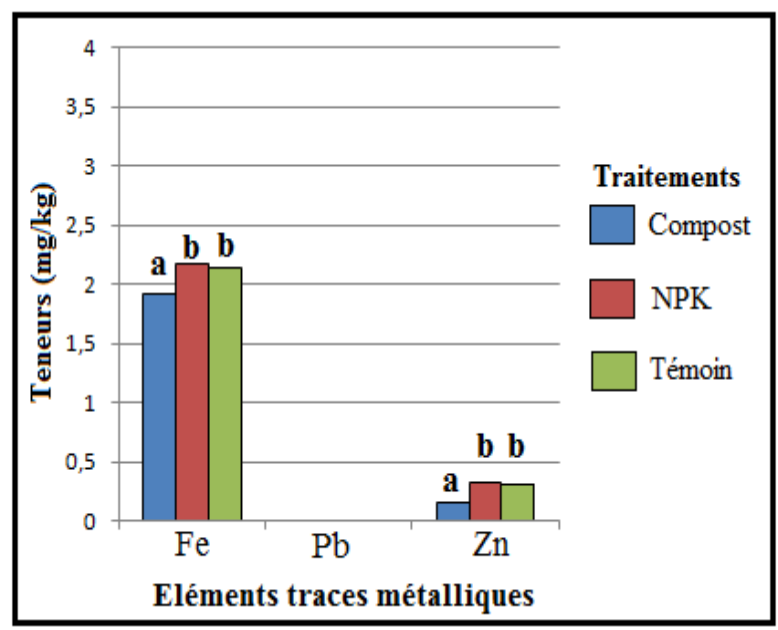

Les lettres a et b désignent pour chaque traitement les valeurs moyennes significativement différentes au seuil de $5 \%$

Figure 10: Teneurs en éléments traces métalliques $(\mathrm{Fe}, \mathrm{Pb}, \mathrm{Zn})$ des récoltes de laitue en fonction des modes de fertilisation. 


\section{DISCUSSION}

\section{Valeur fertilisante du compost utilisé}

Les teneurs en matières organiques sont moyennes $(12,15 \%)$ et basses pour l'azote $(0,65 \%) \quad \mathrm{du}$ compost utilisé comparativement à la norme AFNOR qui a fixé ces seuils, dans les composts en général, à $20 \%$ au moins et $3 \%$ au plus, respectivement pour la matière organique et l'azote (Compaoré et al., 2010). Les déchets ménagers ayant servi à la fabrication du compost utilisé sont riches en composés de nature organique si l'on se réfère aux travaux de Sims (1990). En effet, selon cet auteur, la valeur fertilisante d'un compost de déchets urbains solides est tributaire de la teneur initiale en éléments majeurs des déchets qui ont servi à le fabriquer. Aussi, son rapport $\mathrm{C} / \mathrm{N}$ égal à 10,84, est-il faible, selon la norme AFNOR, qui a situé cette valeur entre 15 et 20 (Compaoré et al., 2010). Toutefois, cette faible valeur du rapport $\mathrm{C} / \mathrm{N}$ est le signe d'un bon niveau de maturité du compost (Nanéma, 2007), c'est-à-dire, un compost presque minéralisé. Cela explique les teneurs relativement élevées de ce compost en éléments basiques $\left(\mathrm{Ca}^{2+}, \mathrm{Mg}^{2+}, \mathrm{K}^{+}\right.$et $\left.\mathrm{Na}^{+}\right)$ nécessaires au bon développement des plantes. Par ailleurs, les teneurs en éléments traces métalliques, notamment le fer et le zinc qui sont avérées faibles dans le compost utilisé constituent, selon les normes standards dans les sols agricoles (Baize 2000), une plusvalue à la qualité de ce compost. Ces normes sont de 140 et $36 \mathrm{mg} \cdot \mathrm{kg}^{-1}$ de sol pour la législation néerlandaise et 150 et $40 \mathrm{mg} \cdot \mathrm{kg}^{-1}$ de sol pour la législation suisse, respectivement pour le fer et le zinc.

Il s'est aussi avéré que le $\mathrm{pH}$ du compost utilisé, égal à 7,3, est ainsi alcalin à l'image du $\mathrm{pH}$ des composts en général. En effet, Steger et al. (2006), qui ont enregistré une augmentation de $\mathrm{pH}$ de 5,4 à 8,5 au bout de 57 semaines de compostage de déchets verts mélangés à des déchets ménagers, ont conclu que le processus de compostage s'accompagne toujours d'un phénomène d'alcalinisation. Ainsi, du fait de leur basicité (Nacro et al., 2010), les composts, en général, sont susceptibles de conférer des propriétés inhibitrices de l'acidité du sol, qui est un frein au développement de la vie dans le sol.

En somme, le compost utilisé a une valeur fertilisante certaine (Tchabi et al., 2012). Aussi, s'il avait été fabriqué dans des conditions idoines (tri des ordures avant leur mise en compostage sous conditions contrôlées), sa valeur fertilisante serait-elle encore plus intéressante (Ayari et al., 2008).

Impacts du compost utilisé sur le rendement de la laitue (Lactuca sativa L.)

Les fertilisants minéraux dosés dans le compost utilisé et jugés favorables dans l'ensemble se sont reflétés aussi sur le rendement de la culture de la laitue mise en place. C'est la preuve que ce compost a amélioré les propriétés du sol utilisé. En effet, Lee et Park (2003) qui ont étudié également la croissance de la laitue en présence de différentes concentrations d'un compost d'ordures ménagères avaient observé, au bout des six premières semaines du cycle de développement de la plante, une croissance des plantes deux à trois fois plus importante en présence de compost par rapport au témoin. Selon ces auteurs, l'effet positif du compost sur la croissance végétale était dû principalement à l'amélioration de la qualité physico-chimique et biologique du sol, du rythme de diffusion des nutriments et de la capacité de rétention d'eau du sol. De plus, les travaux réalisés par Biaou et al. (2017) sur sol ferralitique au Bénin ont montré que l'apport de fertilisants organiques a amélioré la production et le rendement de la carotte (Daucus carota L.). Ainsi, plus le sol est pourvu en matières organiques, plus il devient fertile et productif. La dose de compost de 20 t/ha appliquée par les producteurs de maraîchers de la ville de Daloa devrait donc être revue à la hausse.

Impacts du compost utilisé sur la qualité chimique de la laitue (Lactuca sativa $L$.)

Dans tous les cas, les teneurs en éléments majeurs trouvées dans les récoltes sont restées dans la fourchette des valeurs retrouvées en général dans les plantes (Lerot 2006) : 2 à 4 pour l'azote ; 1 à 2 pour le calcium ; 0,1 à 0,7 pour le magnésium et 0,2 à 1,5 pour le phosphore). Cela montre la bonne 
qualité chimique des récoltes issues de l'ensemble des trois modes de fertilisation testés. Toutefois, le traitement à base de compost avec lequel on a enregistré les teneurs les plus élevées en éléments majeurs notamment en potassium est celui-là même qui a induit à la laitue un effet qualitatif plus important. En effet, au plan chimique, les plantes de meilleure qualité sont en général celles qui ont une importante quantité en éléments majeurs (Temgoua et al., 2015). C'est le contraire pour les éléments traces métalliques (ETM) qui sont réputés toxiques lorsque leurs teneurs dans les sols et, ensuite, dans les récoltes dépassent certains seuils.

Ainsi, l'accumulation de ces éléments dans les sols et les récoltes est devenue une préoccupation majeure en production agricole en raison de leurs effets néfastes sur la croissance des cultures, la qualité des produits alimentaires et la santé de l'environnement (Redon, 2009). Dans la présente recherche, la teneur en fer observée dans les récoltes provenant du traitement témoin $(2,144$ $\mathrm{mg} \cdot \mathrm{kg}^{1}{ }^{1}$ ) était légèrement supérieure à celle des récoltes obtenues sur les sols fertilisés avec le NPK (2,171 mg.kg- $\left.{ }^{1}\right)$, qui, à son tour, dépassait la teneur dosée dans les récoltes issues du traitement à base de compost (1,911 mg.kg- $\left.{ }^{1}\right)$. Dans le cas du zinc, les teneurs déterminées ont varié entre 1,55 et 0,256 mg.kg- ${ }^{1}$. Ces teneurs extrêmes ont été observées, dans cet ordre, dans les récoltes obtenues avec le compost et le traitement témoin. Pour le plomb, quel que soit le traitement, il n'a pas été détecté dans les récoltes. Ces constats montrent que le compost utilisé a inhibé la mobilité des ETM dans le sol, ce qui a empêché leur assimilation par les cultures dans les mêmes proportions que le cas des autres traitements, notamment, le traitement témoin.

En général, la variation de $\mathrm{pH}$ est le facteur dont l'action sur la mobilité des métaux est la plus déterminante (Perrono, 1999) : son abaissement dans le sol favorise la mobilité des ETM, notamment par la mise en solution de sels métalliques ou destruction de la phase de rétention (Perrono, 1999). Ici donc, l'apport de compost au sol a relevé sa teneur en matières organiques et par conséquent le $\mathrm{pH}$ du sol. Cette élévation du $\mathrm{pH}$ a provoqué l'immobilisation des ETM par la formation de composés insolubles ou l'accroissement de la capacité d'échange canonique. Les teneurs d'ETM mesurées dans les récoltes obtenues sont donc loin d'atteindre les seuils problématiques de $17 \mathrm{mg} . \mathrm{kg}^{-1}$ pour le zinc (Mench et Baize 2004 ; Temgoua et al., 2015) et $14 \mathrm{mg} \cdot \mathrm{kg}^{-1}$ pour le fer (FAO/OMS 2016). Ce résultat qui n'est pas surprenant vu les faibles teneurs de ces ETM révélées dans le compost utilisé, dénote la bonne qualité chimique de la laitue produite à Daloa lorsque les producteurs appliquent régulièrement la dose de 20 t/ha de déchets ménagers compostés à l'air pour amender leurs sols. La question est de savoir ce qu'il en sera lorsqu'ils appliqueront des doses de plus en plus grandes de ce type de compost, puisque celle de 20 t/ha appliquée actuellement n'est pas optimale (Zro et al., 2017a).

\section{Conclusion}

Cette étude consistait essentiellement à savoir si l'utilisation des déchets ménagers compostés à l'air libre pour amender les sols, telle que pratiquée par les producteurs de maraîchers de la ville de Daloa (Côte d'Ivoire) a un intérêt sur les plans agronomique et sanitaire. Il en est ressorti que le compost de déchets ménagers a effectivement une valeur fertilisante des sols. En outre, ce compost a été capable d'inhiber la mobilité des ETM dans le sol, les empêchant ainsi d'être assimilés massivement par les cultures. Il en résulte que les laitues produites sont d'une bonne qualité chimique.

Mais, la présence de certains éléments traces métalliques dans la laitue produite avec le compost de déchets ménagers solides compostés à l'air libre dans les décharges, interpelle. Aussi, apparaît-il la nécessité de fabriquer du compost à base d'ordures ménagères préalablement triées, afin de réduire les risques de contamination.

\section{CONFLITS D'INTERETS}

Les auteurs déclarent n'avoir aucun conflit d'intérêt. 


\section{CONTRIBUTIONS DES AUTEURS}

$\mathrm{KZ}$, AADH et SDF ont participé à la conduite des travaux sur le terrain, à la conception, à la rédaction et à la mise en forme de l'article ainsi qu'aux analyses statistiques des données ; Y-KA a supervisé la conduite des travaux, la conception, l'élaboration et la rédaction du manuscrit.

\section{REMERCIEMENTS}

Les auteurs remercient les responsables de l'Université Jean Lorougnon Guédé de Daloa pour leur soutien matériel à l'élaboration de cette étude. Ils remercient également les producteurs de maraichers de la ville de Daloa pour avoir accepté de mettre à leur disposition leurs parcelles pour la conduite de l'étude.

\section{REFERENCES}

Atidegla SC, Agbossou EK, Huat J, Kakai RG. 2011. Contamination métallique des légumes des périmètres maraîchers urbains et péri urbains : Cas de la commune de Grand-Popo au Bénin. Int. J. Biol. Chem. Sci. 5(6): 2351-2361. DOI : http://dx.doi.org/10.4314/ijbcs.v5i6.15.

Avenard JM. 1971. Aspect de la géomorphologie In : Milieu naturel de Côte d'Ivoire. Mémoire ORSTOM, Paris, France, 50 : 8-73.

Ayari F, Gharbi N, Kosai R, Jedidi N. 2008. Effet du tri sélectif sur la contamination par les métaux lourds des composts d'ordures ménagères. Revue Francophone d'Ecologie Industrielle, $52: 4-7$.

Baize D. 2000. Guide des Analyses en Pédologie: Choix, Expression, Présentation, Interprétation. (2 ${ }^{\mathrm{ème}}$ Edn). INRA : Paris, p. 257

Biaou ODB, Saïdou A, Bachabi FX, Padonou GE, Balogoun I. 2017. . Effet de l'apport de différents types d'engrais organiques sur la fertilité du sol et la production de la carotte (Daucus carota L.) sur sol ferralitique au sud Bénin. Int. J. Biol. Chem. Sci. 11(5): 2315-2326. DOI : https://dx.doi.org/10.4314/ijbcs.v11i5.29

Bonhomme R, Ruget F, Derieux M, Vincourt P. 1982. Relations entre production de matière sèche et énergie interceptée chez différents génotypes de maïs. CR Acad. Sc. Paris, série III, 294 : 393-398.

Bricas N, Seck PA. 2004. L'alimentation des villes du Sud : les raisons de craindre et d'espérer. Cahiers Agricultures, 13(1): 10-14.

Brou YT. 2010. Variabilité climatique, déforestation et dynamique agrodémographique en Côte d'Ivoire. Sécheresse, 21(1):1-6. Doi : 10.1684/sec.2010.0277.

Brou YT. 2005. Climat, mutations socioéconomiques et paysages en Côte d'Ivoire. Mémoire de synthèse des activités scientifiques. Habilitation à Diriger des Recherches, Université des Sciences et Technologies de Lille, France, p. 212.

Chabalier PF, Van de Kerchove V, Saint Macary H. 2006.Guide de la fertilisation organique à la Réunion. CIRAD, p. 166.

Compaoré E, Nanéma LS, Bonkoungou S, Sedogo MP. 2010. Evaluation de la qualité de compost de déchets urbains solides de la ville de Bobo-Dioulasso, Burkina-Faso pour une utilisation efficiente en agriculture. Journal of applied biosciences, 33 : 2076-2083.

Dabin B, Leneuf N, Riou G. 1960. Carte pédologique de la Côte d'Ivoire au 1/2.000.000. Notice explicative ORSTOM, p. 39.

Dagnelie P. 2008. Le plan d'expérience évolue. Revue MODULAD, 38: 13-36.

Djogbédé AZK, Hinvi LC, Fiogbé ED. 2012. Effets de substitution des engrais chimiques par Azolla pinnata en riziculture au Nord Bénin. Int. J. Biol. Chem. Sci 6(6): 3027-3044. DOI : http://dx.doi.org/10.4314/ijbcs.v6i6.5 
Dragon S, Icard C. 2010. Effet d'apport de différents amendements organiques sur les propriétés du sol - Bilan de 15 années d'essai en culture légumière à la SERAIL. Echo-MO., 81 : 4-8.

FAO/OMS (2016). Programme mixte FAO/OMS sur les normes alimentaires commission du codex alimentarius. Trente-neuvième session, Rome, Italie, p. 16.

France-Ida J. 1992. Analyse de produits naturels de taxus canadensis. Mémoire présenté comme exigence partielle de la maîtrise en ressources renouvelables. Université du Québec, p. 104.

Houot S. 2005. Recyclage de déchets sur les sols : valeur agronomique et impacts environnementaux. In Sols et Environnement, Girard MC, Walter C, Remy JC, Berthelin J, Morel JL (eds). Dunod : Paris ; 262-284.

LANO. 2008. Analyses des terres. http://www.lano.asso.fr/web/analyses.ht $\mathrm{ml}$ [Consulté en mai 2017].

Lee JJ, Park RD. 2003. Effet of wast compost on microbial population soil enzyme activity and lettuce growth. Bioresources technology, 93(1) : 21-28. https://www.tandfonline.com/doi/full/.../ 00103624.2014.884103?

Lerot B. 2006. Les éléments minéraux, http://www.orchidstory.be/site/mineraux. pdf. Consulté le 20/08/2016.

Lesafre B. 2004. L'alimentation des villes : un nouveau défi pour la recherche. Cahiers Agricultures, 13(1): 10-14. Amsterdam: SPB Academic Publishing, 757-766. http://dx.doi.org/10.1007/978-94-0178745-1_12
Mench M, Baize D. 2004. Contamination des sols et de nos aliments d'origine végétale par les éléments en traces : mesures pour réduire l'exposition. Courrier de l'Environnement de l'INRA, 52 : 26.

Nacro S, Ouédraogo S, Traoré K, Sankara E, Kaboré C, Ouattara B. 2010. Effets comparés des pratiques paysannes et des bonnes pratiques agricoles de gestion de la fertilité des sols sur les propriétés des sols et les rendements des cultures dans la zone sud soudanienne du Burkina Faso. Int. J. Biol. Chem. Sci., 4(4): 1044-1055.

Nanéma SL. 2007.Compostage et évaluation de l'efficacité agronomique du compost de déchets urbains solides de la ville de Bobo-Dioulasso, mémoire d'ingénieur, IPR/IFRA, Katiébougou (Mali), p. 67.

Perrono P. 1999. Les micropolluants métalliques des boues de stations d'épuration urbaine et l'épandage agricole. Mém. D.U.E.S.S., $\quad$ D.E.P., Univ. Picardie, Amiens, p. 62.

Redon P-O. 2009. Rôle de champignons mycorhiziens à arbuscules dans le transfert du cadmium $(\mathrm{Cd})$ du sol à la luzerne (Medicago truncatula). Thèse de Doctorat, Université Henri Poincaré, Nancy, p. 182.

Remon E, Bouchardon J-L, Joly J, Cornier BFaure O. 2009. Accumulation et effets des éléments métalliques sur les populations végétales spontanées de trois crassiers métallurgiques : Peut-on utiliser les plantes comme bioindicateurs? Étude et Gestion des Sols, 16(3/4): 313-321.

Sims JT. 1990. Nitrogen mineralization and elemental availability in soils amended 
with composted sewage sludge. $J$. Environ. Qual., 19: 669-675. DOI: $10.2134 /$ jeq1990.0047242500190004000 $7 \mathrm{x}$.

Steger MF, Frazier P, Oishi S, Kaler M. 2006.

The Meaning of Life Questionnaire: assessing then presence of and search for meaning in life. Journal of Counseling Psychology, 53: 80-93. DOI: 10.1037/0022-0167.53.1.80.80.

Tchabi VI, Azocli D, Biaou GD. 2012. Effet de différentes doses de bouse de vache sur le rendement de la laitue (Lactuca sativa L.) à Tchatchou au Bénin. Int. J. Biol. Chem. Sci., 6(6): 5078-5084. DOI : http://dx.doi.org/10.4314/ijbcs.v6i6.26.

Temgoua E, Tsafack HN, Pfeifer H-R, Njine T. 2015. Teneurs en éléments majeurs et oligoéléments dans un sol et quelques cultures maraîchères de la ville de Dschang, Cameroun. African Crop Science Journal, 23 (1): 35-44.
Zro BGF, Abobi AH, Guéi AM, Soro D, Bakayoko S, Yao-Kouamé A. 2017a. Impacts des apports de résidus ménagers compostés à l'air libre sur l'évolution de la fertilité chimique d'un sol sableux utilisé en maraîchage à Daloa (Côte d'Ivoire). Revue CAMES, sous presse.

Zro BGF, Guéi AM, Konaté Z, Soro D, Bakayoko S, Yao-Kouamé A. 2017b. Impacts of soil fertilization based on fresh bovine dung on the quality of lettuce (Lactuca sativa L.) produced in the market agrosystems of the town of Daloa (Côte d'Ivoire). International Journal of Development Research, Vol. 07(5): 12930-12939.

Zro BGF, Guéi AM, Nangah KY, Soro D, Bakayoko S. 2016. Statistical approach to the analysis of the variability and fertility of vegetable soils of Daloa (Côte d'Ivoire). African Journal of Soil Science, 4(4): 328-338. 